\title{
Quantum Mechanics at Planck's scale and Density Matrix
}

\author{
A.E.Shalyt-Margolin ${ }^{*}$ J.G.Suarez ${ }^{\dagger}$ \\ National Center of Particles and High Energy Physics, Bogdanovich Str. 153, Minsk \\ 220040, Belarus \\ PACS : $03.65 ; 05.30$ \\ Keywords: fundamental length, general uncertainty relations, density matrix, \\ deformed Liouville's equation
}

\begin{abstract}
In this paper Quantum Mechanics with Fundamental Length is chosen as Quantum Mechanics at Planck's scale. This is possible due to the presence in the theory of General Uncertainty Relations. Here Quantum Mechanics with Fundamental Length is obtained as a deformation of Quantum Mechanics. The distinguishing feature of the proposed approach in comparison with previous ones, lies on the fact that here density matrix subjects to deformation whereas so far commutators have been deformed. The density matrix obtained by deformation of quantum-mechanical density one is named throughout this paper density pro-matrix. Within our approach two main features of Quantum Mechanics are conserved: the probabilistic interpretation of the theory and the well-known measuring procedure corresponding to that interpretation. The proposed approach allows to describe dynamics. In particular, the explicit form of deformed Liouville's equation and the deformed Shrödinger's picture are given. Some implications of obtained results are discussed. In particular, the problem of singularity, the hypothesis of cosmic censorship, a possible improvement of the definition of statistical entropy and the problem of information loss in black holes are considered. It is shown that obtained results allow to deduce in a simple
\end{abstract}

*Phone (+375) 172 883438; e-mail: a.shalyt@mail.ru; alexm@hep.by

†Phone (+375) 172 883438; e-mail: suarez@hep.by, jsuarez@mail.tut.by 
and natural way the Bekenstein-Hawking's formula for black hole entropy in semiclassical approximation.

\section{Introduction}

In this paper Quantum Mechanics with Fundamental Length (QMFL) is considered as Quantum Mechanics (QM) of the early Universe. The main motivation for this choice is the presence in the theory of General Uncertainty Relations (GUR) appropriated to describe the physical behavior of the early Universe and unavoidable conducting to the concept of fundamental length. Here QMFL is obtained as a deformation of QM, choosing the quantity $\beta=l_{\text {min }}^{2} / x^{2}$ (where $x$ is the scale) as the parameter of deformation of the theory. The main difference between used approach and previous ones lies on the fact, that we propose to deform density matrix whereas so far commutators have been deformed. Obtained in such a way density matrix (generalized density matrix) is called here and throughout this paper density pro-matrix. Within our approach two very important features of QM have been conserved in QMFL. Namely, the probabilistic interpretation and the well-known measuring procedure, corresponding to this interpretation. It was shown that in the paradigm of expanding model of the Universe there are two different (unitary non-equivalent) Quantum Mechanics: the first one named QMFL is describing nature at Planck's scale or on the early Universe and it is based on GUR. The second one named QM and representing passage to the limit from Planck's to low energy scale is based on Heisenberg's Uncertainty Relations (UR). Consequently, some well-known quantum mechanical concepts could appear only in the low energy limit. Further, within the proposed approach some dynamical aspects of QMFL are described. In particular, a deformation of the Liouville's equation, the Shrödinger's picture in QMFL as well as some implications of obtained results are presented. Mentioned implications deal with the problem of singularity, the hypothesis of cosmic censorship, a possible improvement of definition of statistical entropy and also with the problem of information loss in black holes. The Bekenstein-Hawking's

formula for black hole entropy in semiclassical approximation is deduced within proposed approach in a simple and natural way. 


\section{Fundamental Length and Density Matrix}

Using different approaches (String Theory [2], Gravitation [3], Quantum Theory of black holes [4 etc.) the authors of numerous papers issued over the last 14-15 years have pointed out that Heisenberg's Uncertainty Relations should be modified. Specifically, a high energy correction has to appear

$$
\triangle x \geq \frac{\hbar}{\triangle p}+\alpha L_{p}^{2} \frac{\triangle p}{\hbar}
$$

Here $L_{p}$ is the Planck's length: $L_{p}=\sqrt{\frac{G \hbar}{c^{3}}} \simeq 1,610^{-35} m$ and $\alpha>0$ is a constant. In [3] it was shown that this constant may be chosen equal to 1. However, here we will use $\alpha$ as an arbitrary constant without giving it any definite value. The inequality (1) is quadratic in $\triangle p$ :

$$
\alpha L_{p}^{2}(\triangle p)^{2}-\hbar \triangle x \triangle p+\hbar^{2} \leq 0
$$

from whence the fundamental length is

$$
\triangle x_{\min }=2 \sqrt{\alpha} L_{p}
$$

Since in what follows we proceed only from the existence of fundamental length, it should be noted that this fact was established apart from GUR as well. For instance, from an ideal experiment associated with Gravitational Field and Quantum Mechanics a lower bound on minimal length was obtained in 6], 7] and improved in 8] without using GUR to an estimate of the form $\sim L_{p}$. Let us to consider equation (3) in some detail. Squaring both its sides, we obtain

$$
\left(\overline{\Delta \widehat{X}^{2}}\right) \geq 4 \alpha L_{p}^{2}
$$

Or in terms of density matrix

$$
S p\left[\left(\rho \widehat{X}^{2}\right)-S p^{2}(\rho \widehat{X})\right] \geq 4 \alpha L_{p}^{2}=l_{\text {min }}^{2}>0,
$$

where $\widehat{X}$ is the coordinate operator. Expression (5) gives the measuring rule used in QM. However, in the case considered here, in comparison with $\mathrm{QM}$, the right part of (15) cannot be done arbitrarily near to zero since it is limited by $l_{\min }^{2}>0$, where due to GUR $l_{\min } \sim L_{p}$.

Apparently, this may be due to the fact that QMFL with GUR (11) is unitary non-equivalent to QM with UR. Actually, in QM the left-hand 
side of (15) can be chosen arbitrary closed to zero, whereas in QMFL this is impossible. But if two theories are unitary equivalent then, the form of their spurs should be retained. Besides, a more important aspect is contributing to unitary non-equivalence of these two theories: QMFL contains three fundamental constants (independent parameters) $G, c$ and $\hbar$, whereas QM contains only one: $\hbar$. Within an inflationary model (see 9]), QM is the low-energy limit of QMFL (QMFL turns to QM) for the expansion of the Universe. In this case, the second term in the right-hand side of (11) vanishes and GUR turn to UR. A natural way for studying QMFL is to consider this theory as a deformation of QM, turning to QM at the low energy limit (during the expansion of the Universe after the Big Bang). We will consider precisely this option. However differing from authors of papers [4], [5] and others, we do not deform commutators, but density matrix, leaving at the same time the fundamental quantum-mechanical measuring rule (5) without changes. Here the following question may be formulated: how should be deformed density matrix conserving quantum-mechanical measuring rules in order to obtain self-consistent measuring procedure in QMFL? For answering to the question we will use the R-procedure. For starting let us to consider R-procedure both at the Planck's energy scale and at the low-energy one. At the Planck's scale $a \approx i l_{\text {min }}$ or $a \sim i L_{p}$, where $i$ is a small quantity. Further $a$ tends to infinity and we obtain for density matrix

$$
S p\left[\rho a^{2}\right]-S p[\rho a] S p[\rho a] \simeq l_{\min }^{2} \text { or } S p[\rho]-S p^{2}[\rho] \simeq l_{\min }^{2} / a^{2} .
$$

Therefore:

1. When $a<\infty, S p[\rho]=S p[\rho(a)]$ and $S p[\rho]-S p^{2}[\rho]>0$. Then, $S p[\rho]<1$ that corresponds to the QMFL case.

2. When $a=\infty, S p[\rho]$ does not depend on $a$ and $S p[\rho]-S p^{2}[\rho] \rightarrow 0$. Then, $S p[\rho]=1$ that corresponds to the QM case.

How should be points 1 and 2 interpreted? How does analysis above-given agree to the main result from [29] ${ }^{1}$ ? It is in full agreement. Indeed, when state-vector reduction (R-procedure) takes place in QM then, always an eigenstate (value) is chosen exactly. In other words, the probability is equal

\footnotetext{
$1 " . .$. there cannot be any physical state which is a position eigenstate since a eigenstate would of course have zero uncertainty in position"
} 
to 1 . As it was pointed out in the above-mentioned point 1 the situation changes when we consider QMFL: it is impossible to measure coordinates exactly since it never will be absolutely reliable. We obtain in all cases a probability less than $1(S p[\rho]=p<1)$. In other words, any R-procedure in QMFL leads to an eigenvalue, but only with a probability less than 1. This probability is as near to 1 as far the difference between measuring scale $a$ and $l_{\min }$ is growing, or in other words, when the second term in (11) becomes insignificant and we turn to QM. Here there is not a contradiction with [29]. In QMFL there are not exact coordinate eigenstates (values) as well as there are not pure states. In this paper we do not consider operator properties in QMFL as it was done in [29] but density-matrix properties.

The properties of density matrix in QMFL and QM have to be different. The only reasoning in this case may be as follows: QMFL must differ from QM, but in such a way that in the low-energy limit a density matrix in QMFL must coincide with the density matrix in QM. That is to say, QMFL is a deformation of QM and the parameter of deformation depends on the measuring scale. This means that in QMFL $\rho=\rho(x)$, where $x$ is the scale, and for $x \rightarrow \infty \rho(x) \rightarrow \widehat{\rho}$, where $\widehat{\rho}$ is the density matrix in QM.

Since on the Planck's scale $S p[\rho]<1$, then for such scales $\rho=\rho(x)$, where $x$ is the scale, is not a density matrix as it is generally defined in QM. On Planck's scale we name $\rho(x)$ "density pro-matrix". As follows from the above, the density matrix $\widehat{\rho}$ appears in the limit

$$
\lim _{x \rightarrow \infty} \rho(x) \rightarrow \widehat{\rho}
$$

when GUR (II) turn to UR and QMFL turns to QM.

Thus, on Planck's scale the density matrix is inadequate to obtain all information about the mean values of operators. A "deformed" density matrix (or pro-matrix) $\rho(x)$ with $S p[\rho]<1$ has to be introduced because a missing part of information $1-S p[\rho]$ is encoded in the quantity $l_{\min }^{2} / a^{2}$, whose specific weight decreases as the scale $a$ expressed in units of $l_{\min }$ is going up.

\section{QMFL as a deformation of QM}

Here we describe QMFL as a deformation of QM using the above-developed formalism of density pro-matrix. Within it density pro-matrix should be 
understood as a deformed density matrix in QMFL. As fundamental parameter of deformation we use the quantity $\beta=l_{\min }^{2} / x^{2}$, where $x$ is the scale.

\section{Definition 1.}

Any system in QMFL is described by a density pro-matrix of the form $\rho(\beta)=\sum_{i} \omega_{i}(\beta)|i><i|$, where

1. $0<\beta \leq 1 / 4$

2. Vectors $\mid i>$ form a full orthonormal system;

3. Coefficients $\omega_{i}(\beta) \geq 0$ and for all $i$ the limit $\lim _{\beta \rightarrow 0} \omega_{i}(\beta)=\omega_{i}$ exists;

4. $S p[\rho(\beta)]=\sum_{i} \omega_{i}(\beta)<1, \sum_{i} \omega_{i}=1$;

5. For every operator $B$ and any $\beta$ there is a mean operator $B$ depending on $\beta$ :

$$
<B>_{\beta}=\sum_{i} \omega_{i}(\beta)<i|B| i>
$$

Finally, in order that our definition 1 agrees to the result of section 2 , the following condition must be fulfilled:

$$
S p[\rho(\beta)]-S p^{2}[\rho(\beta)] \approx \beta
$$

Hence we can find the value for $S p[\rho(\beta)]$ satisfying the condition of definition 1:

$$
S p[\rho(\beta)] \approx \frac{1}{2}+\sqrt{\frac{1}{4}-\beta} .
$$

According to point $5<1>_{\beta}=S p[\rho(\beta)]$. Therefore, for any scalar quantity $f$ we have $<f>_{\beta}=f S p[\rho(\beta)]$. In particular, the mean value $<\left[x_{\mu}, p_{\nu}\right]>_{\beta}$ is equal to

$$
<\left[x_{\mu}, p_{\nu}\right]>_{\beta}=i \hbar \delta_{\mu, \nu} S p[\rho(\beta)] .
$$

We denote the limit $\lim _{\beta \rightarrow 0} \rho(\beta)=\rho$ as the density matrix. Evidently, in the limit $\beta \rightarrow 0$ we return to QM.

As follows from definition $1,<|j><j|>_{\beta}=\omega_{j}(\beta)$, from whence the completeness condition by $\beta$ is 
$<\left(\sum_{i}|i><i|\right)>_{\beta}=<1>_{\beta}=S p[\rho(\beta)]$. The norm of any vector $\mid \psi>$ assigned to $\beta$ can be defined as

$$
<\psi\left|\psi>_{\beta}=<\psi\right|\left(\sum_{i}|i><i|\right)_{\beta}|\psi>=<\psi|(1)_{\beta}|\psi>=<\psi| \psi>S p[\rho(\beta)],
$$

where $\langle\psi \mid \psi\rangle$ is the norm in QM, i.e. for $\beta \rightarrow 0$. Similarly, the described theory may be interpreted using a probabilistic approach, however requiring replacement of $\rho$ by $\rho(\beta)$ in all formulae.

It should be noted:

I. The above limit covers both Quantum and Classical Mechanics. Indeed, since $\beta \sim L_{p}^{2} / x^{2}=G \hbar / c^{3} x^{2}$, we obtain:

a. $(\hbar \neq 0, x \rightarrow \infty) \Rightarrow(\beta \rightarrow 0)$ for $\mathrm{QM}$;

b. $(\hbar \rightarrow 0, x \rightarrow \infty) \Rightarrow(\beta \rightarrow 0)$ for Classical Mechanics;

II. As a matter of fact, the deformation parameter $\beta$ should assume the value $0<\beta \leq 1$. However, as seen from (8),$S p[\rho(\beta)]$ is well defined only for $0<\beta \leq 1 / 4$. That is if $x=i l_{\text {min }}$ and $i \geq 2$ then, there is not any problem. At the point where $x=l_{\min }$ there is a singularity related to complex values assumed by $S p[\rho(\beta)]$, i.e. to the impossibility of obtaining a diagonalized density pro-matrix at this point over the field of real numbers. For this reason definition 1 has no sense at the point $x=l_{\min }$. We will come back to the question appearing in this section when we will discuss singularity and hypothesis of cosmic censorship in section 5 .

III. We consider possible solutions for (77). For instance, one of the solutions of (7), at least to the first order in $\beta$, is

$$
\rho^{*}(\beta)=\sum_{i} \alpha_{i} \exp (-\beta)|i><i|,
$$

where all $\alpha_{i}>0$ are independent of $\beta$ and their sum is equal to 1 . In this way $S p\left[\rho^{*}(\beta)\right]=\exp (-\beta)$. Indeed, we can easily verify that

$$
S p\left[\rho^{*}(\beta)\right]-S p^{2}\left[\rho^{*}(\beta)\right]=\beta+O\left(\beta^{2}\right) .
$$

The exponential ansatz for $\rho^{*}(\beta)$ given here will be taking into account in further sections. Note that in the momentum representation 
$\beta=p^{2} / p_{p l}^{2}$, where $p_{p l}$ is the Planck's momentum. When present in matrix elements, $\exp (-\beta)$ can damp the contribution of great momenta in a perturbation theory.

IV. It is clear that within the proposed description the states with a unit probability, i.e. pure states, can appear only in the limit $\beta \rightarrow 0$, when all $\omega_{i}(\beta)$ except one are equal to zero or when they tend to zero at this limit. In our treatment pure states are states, which can be represented in the form $|\psi\rangle<\psi \mid$, where $\langle\psi \mid \psi\rangle=1$.

V. We suppose that all definitions concerning a density matrix can be carry over to the above-mentioned deformation of Quantum Mechanics (QMFL) changing the density matrix $\rho$ by the density pro-matrix $\rho(\beta)$ and subsequent passage to the low-energy limit $\beta \rightarrow 0$. Specifically, for statistical entropy we have

$$
S_{\beta}=-S p[\rho(\beta) \ln (\rho(\beta))] .
$$

The quantity of $S_{\beta}$ seems never to be equal to zero as $\ln (\rho(\beta)) \neq 0$ and hence $S_{\beta}$ may be equal to zero at the limit $\beta \rightarrow 0$ only.

Some Implications:

I. If we carry out a measurement at a pre-determined scale, it is impossible to regard the density pro-matrix as a density matrix with an accuracy better than the limit $\sim 10^{-66+2 n}$, where $10^{-n}$ is the measuring scale. In the majority of known cases this is sufficient to consider the density pro-matrix as a density matrix. But at Planck's scale, where quantum gravitational effects and Planck's energy levels cannot be neglected, the difference between $\rho(\beta)$ and $\rho$ should be taken into consideration.

II. Proceeding from the above, on Planck's scale the notion of Wave Function of the Universe (as introduced in [10]) has no sense, and quantum gravitation effects in this case should be described with the help of density pro-matrix $\rho(\beta)$ only.

III. Since density pro-matrix $\rho(\beta)$ depends on the measuring scale, evolution of the Universe within the inflationary model paradigm [9] is not a unitary process, or otherwise the probabilities $p_{i}=\omega_{i}(\beta)$ would be preserved. 


\section{Dynamical aspects of QMFL}

Let's suppose that in QMFL density pro-matrix has the form $\rho[\beta(t), t]$ or in other words, it depends on two parameters: time $t$ and parameter of deformation $\beta$, which also depends on time $(\beta=\beta(t))$. Then, we have

$$
\rho[\beta(t), t]=\sum \omega_{i}[\beta(t)]|i(t)><i(t)| .
$$

Differentiating the last expression on time we obtain the equation

$$
\frac{d \rho}{d t}=\sum_{i} \frac{d \omega_{i}[\beta(t)]}{d t}|i(t)><i(t)|-i[H, \rho(\beta)]=d[\ln \omega(\beta)] \rho(\beta)-i[H, \rho(\beta)] \text {. }
$$

Where $\ln [\omega(\beta)]$ is a row-matrix and $\rho(\beta)$ is a column-matrix. In such a way we have obtained a prototype of the Liouville's equation.

Let's consider some particular cases of importance.

I. First we consider the process of time evolution at low energies, or in other words, when $\beta(0) \approx 0, \beta(t) \approx 0$ and $t \rightarrow \infty$. Then it is clear that $\omega_{i}(\beta) \approx \omega_{i} \approx$ constant. The first term in (13) vanishes and we obtain the Liouville's equation.

II. We obtain also the Liouville's equation when we turn from inflationary to large-scale. Within the inflationary approach the scale $a \approx e^{H t}$, where $H$ is the Hubble's constant and $t$ is time. Therefore $\beta \sim e^{-2 H t}$ and when $t$ is quite big $\beta \rightarrow 0$. In other words, $\omega_{i}(\beta) \rightarrow \omega_{i}$, the first term in (13) vanishes and we obtain again the Liouville's equation.

III. At very early inflationary-process stage or even before it takes place $\omega_{i}(\beta)$ was not a constant and therefore, the first term in (13) should be taking into account. This way we obtain a deviation from the Liouville's equation.

IV. At last, let us consider the case when $\beta(0) \approx 0, \beta(t)>0$ where $t \rightarrow \infty$. In this case we are going from low-energy scale to high one and $\beta(t)$ grows when $t \rightarrow \infty$. In this case the first term in (13) is significant and we obtain an addition to the Liouville's equation in the form

$$
d[\ln \omega(\beta)] \rho(\beta) .
$$

This case could take place when matter go into a black hole and is moving in direction of the singularity (to the Planck's scale). 


\section{Singularity, Entropy and Information Loss in Black Holes}

It follows to note that remark II in section 3 about complex meaning assumed by density pro-matrix at the point with fundamental length has straightforward relation with the singularity problem and cosmic censorship in General Theory of Relativity [11]. Indeed, considering, for instance, a Schwarzchild's black hole ([12]) with metrics:

$$
d s^{2}=-\left(1-\frac{2 M}{r}\right) d t^{2}+\frac{d r^{2}}{\left(1-\frac{2 M}{r}\right)}+r^{2} d \Omega_{I I}^{2},
$$

we obtain, as it is well-known a singularity at the point $r=0$. In our approach this corresponds to the point with fundamental length $\left(r=l_{\text {min }}\right)$. At this point we are not able to measure anything, since at this point $\beta=1$ and $S p[\rho(\beta)]$ becomes complex. Thus, we carry out a measurement, starting from the point $r=2 l_{\text {min }}$ corresponding to $\beta=1 / 4$. Consequently, the initial singularity $r=l_{\text {min }}$, which cannot be measured, is hidden of observation. This could confirm the hypothesis of cosmic censorship in this concrete case. This hypothesis claims that "a naked singularity cannot be observed". Thus, QMFL in our approach feels the singularity. (In comparison with QM, which does not feel it).

Statistical entropy, connected with density pro-matrix and introduced in the remark $\mathrm{V}$, section 3

$$
S_{\beta}=-S p[\rho(\beta) \ln (\rho(\beta))],
$$

may be interpreted as density of entropy on unity of minimal square $l_{\min }^{2}$ depending on the scale $x$. It could be quite big nearby the singularity. In other words, when $\beta \rightarrow 1 / 4$. This does not contradict the second law of Thermodynamics since the maximal entropy of a determined object in the Universe is proportional to the square of their surface $A$, measured in units of minimal square $l_{\text {min }}^{2}$ or Planck's square $L_{p}^{2}$, as it was shown in some papers (see, for instance [13). Therefore, in the expanded Universe since surface $A$ grows, then entropy does not decrease.

Obtained results enable one to consider anew the information loss problem associated with black holes [14, 15, at least, for the case of "mini" black holes. Indeed, when we consider these black holes, Planck's scale is a factor. It was shown that entropy of matter absorbed by a black hole 
at this scale is not equal to zero, supporting the data of R.Myers [16]. According to his results, a pure state cannot form a black hole. Then, it is necessary to reformulate the problem per se, since so far in all publications on information paradox zero entropy at the initial state has been compared to nonzero entropy at the final state. According to our analysis at the Planck's scale there is not initial zero entropy and "mini" black holes with masses of the order $M_{p l}$ should not radiate at all. Similar results using another approach were deduced by A.D.Hefler [30]: "p.1... The possibility that non-radiating "mini" black holes should be taken seriously; such holes could be part of the dark matter in the Universe". It follows to note, that in [30], the main argument in favor of the existence of non-radiating "mini" black holes is founded under the consideration of quantum gravity effects. In our analysis these effects are considered implicitly since any approach in quantum gravity leads to the fundamental-length concept [26]. Besides, it should be noted that in some recent papers for all types of black holes QMFL with GUR is considered at the very beginning [17, 31]. As a consequence of this approach, stable remnants with masses of the order of Planck's ones $M_{p l}$ emerge during the process of black hole evaporation. From here it follows that black holes should not evaporate fully. We arrive to the conclusion that results given in [12, 18, are correct only in the semi-classical approximation and they should not be applicable to the quantum back hole analysis. Based on our results, we can elucidate (at least qualitatively) the problem associated with information loss on black holes formed when a star collapses. Actually, near the horizon of events the entropy of an approximately pure state is practically equal to zero: $S^{i n}=-S p[\rho \ln (\rho)]$ that is associated with the value $\beta \mapsto 0$. When approaching the singularity $\beta>0$ (i.e. on Plank's scale), its entropy is nonzero for $S_{\beta}=-S p[\rho(\beta) \ln (\rho(\beta))]$. Therefore, in such a black hole the entropy increases, whereas information is lost.

On the other hand, from the results obtained above, at least at the qualitative level, it can be clear up the answer to the question how may be information lost in big black holes, which are formed as result of star collapse. Our point of view is closed to the R. Penrose's one [19]. He considers that information in black holes is lost when matter meets a singularity. In our approach information loss takes place in the same form. Indeed, near to the horizon of events an approximately pure state with practically equal to zero initial entropy $S^{i n}=-S p[\rho \ln (\rho)]$, which corresponds to $\beta \rightarrow 0$, when approaching a singularity (in other words, is reaching the Planck's 
scale) gives yet non zero entropy $S_{\beta}=-S p[\rho(\beta) \ln (\rho(\beta))]>0$ when $\beta>0$. Therefore, entropy increases and information is lost in this black hole. We can (at the moment, also at the qualitative level) evaluate entropy of black holes. Indeed, starting from density matrix for a pure state at the "entry" of a black hole $\rho_{i n}=\rho_{\text {pure }}$ with zero entropy $S^{i n}=0$, we obtain, doing a straightforward "naive" calculation (this means that (17) is considered as an exact relation). Then, at the singularity in the black hole density pro-matrix $S p\left[\rho_{\text {out }}\right]=1 / 2$ ) for $\beta=1 / 4$ with entropy

$$
S^{\text {out }}=S_{1 / 4}=-1 / 2 \ln 1 / 2 \approx 0.34657 .
$$

Taking into account that total entropy of a black hole is proportional to quantum area of surface A, measured in Planck's units of area $L_{p}^{2}$ [20, we obtain the following value for quantum entropy of a black hole:

$$
S_{B H}=0.34657 \frac{A}{L_{p}^{2}}
$$

This formula differs from the well-known one given by BekensteinHawking for black hole entropy $S_{B H}=\frac{1}{4} \frac{A}{L_{p}^{2}}$ [21]. This result was obtained in the semi-classical approximation. At the present moment quantum corrections to this formula are object of investigation [22. As it was yet above-mentioned we carry out a straightforward calculation. Otherwise, using the ansatz of the remark III in section 3 and assuming that spur of density pro-matrix is equal to $S p\left[\rho^{*}(\beta)\right]=\exp (-\beta)$, we obtain for $\beta=1 / 4$ that entropy is equal to

$$
S^{* o u t}=S_{1 / 4}^{*}=-S p[\exp (-1 / 4) \ln \exp (-1 / 4)] \approx 0.1947,
$$

and consequently we arrive to the value for entropy

$$
S_{B H}=0.1947 \frac{A}{L_{p}^{2}}
$$

that is nearest to the result obtained in [22]. Our approach, leading to formula (16) is at the very beginning based on the quantum nature of black holes. Let us to note here that in the approaches, used up to now to modify Liouville's equation, due to information paradox [23], the added member appearing in the right side of (13) has the form

$$
-\frac{1}{2} \sum_{\alpha \beta \neq 0}\left(Q^{\beta} Q^{\alpha} \rho+\rho Q^{\beta} Q^{\alpha}-2 Q^{\alpha} \rho Q^{\beta}\right),
$$


where $Q^{\alpha}$ is a full orthogonal set of Hermitian matrices with $Q^{0}=1$. In this case either locality or conservation of energy-impulse tensor is broken down. In the offered in this paper approach, the added member in the deformed Liouville's equation has a more natural and beautiful form in our opinion:

$$
d[\ln \omega(\beta)] \rho(\beta) .
$$

In the limit $\beta \rightarrow 0$ all properties of QM are conserved, the added member vanishes and we obtain Liouville's equation.

\section{Bekenstein-Hawking Formula}

Whether can the well-known semiclassical Bekenstein-Hawking formula for Black Hole entropy be obtained within the proposed approach? Let us show how to do it. At the moment our deduction is founded on an heuristic argumentation. However we are sure a more strong argumentation can be provided. To obtain black hole quantum entropy we use the formula $S_{\beta}=-S p[\rho(\beta) \ln (\rho(\beta))]=-<\ln (\rho(\beta))>_{\beta}$ when $\beta$ takes its maximal meaning $(\beta=1 / 4)$. In this case (15) and (16) can be written as

$$
S_{B H}=-<\ln (\rho(1 / 4))>_{1 / 4} \frac{A}{L_{p}^{2}},
$$

for different $\rho(\beta)$ in (15) and (16) but for the same meaning of $\beta(\beta=1 / 4)$. Semiclassical approximation works only at large-scales, therefore measuring procedure is also defined at the large-scales. In other words, all mean values must be taken when $\beta=0$. However, for operators, whose mean values are calculated the dependence on $\beta$ must be taking into account since according to the well-known Hawking's paper 14] operator of superscattering $\$$ translates $\$: \rho_{\text {in }} \mapsto \rho_{\text {out }}$, where in the case considered $\rho_{\text {in }}=\rho_{\text {pure }}$ and $\rho_{\text {out }}=\rho_{\text {pure }}^{*}(\beta)=\exp (-\beta) \rho_{\text {pure }}=\exp (-1 / 4) \rho_{\text {pure }}$ in correspondence with the exponential ansatz of point III, section 3 . Therefore we have

$$
S_{\beta}^{\text {semiclass }}=-<\ln (\rho(\beta))>
$$

and formula for semiclassical entropy for a black hole takes the form

$$
S_{B H}^{\text {semiclass }}=-<\ln (\rho(1 / 4))>\frac{A}{L_{p}^{2}}=-<\ln [\exp (-1 / 4)] \rho_{\text {pure }}>\frac{A}{L_{p}^{2}}=\frac{1}{4} \frac{A}{L_{p}^{2}}
$$


that coincides with the well-known Bekestein-Hawking formula. It follows to note that the meaning $\beta=1 / 4$ in our approach appears in section 3 non in an artificial way but as the maximal meaning for which $S p \rho(\beta)$ still stays real, according to (7) and (8). Apparently, if considering corrections of order higher than 1 on $\beta$, then members from $O\left(\beta^{2}\right)$ in the formula

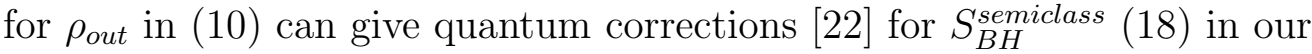
approach.

\section{Some comments on Shrödinger's picture}

A procedure allowing to obtain a theory from the transformation of the precedent one is named "deformation". This is doing, using one or a few parameters of deformation in such a way, that the original theory must appear in the limit, when all parameters tend to some fixed values. The most clear example is QM being a deformation of Classical Mechanics. The parameter of deformation in this case is the Planck's constant $\hbar$. When $\hbar \rightarrow 0$ QM passages to Classical Mechanics. As it was indicated above in the remark 1 section 3, we are able to obtain from QMFL two limits: Quantum and Classical Mechanics. The described here deformation should be understood as "minimal" in the sense that we have deformed only the probability $\omega_{i} \rightarrow \omega_{i}(\beta)$, whereas state vectors have been not deformed. In a most complete consideration we will be obligated to consider instead $|i><i|$, vectors $|i(\beta)><i(\beta)|$ and in this case the full picture will be very complicated. It is easy to understand how Shrodinger's picture is transformed in QMFL. The prototype of Quantum Mechanical normed wave function $\psi(q)$ with $\int|\psi(q)|^{2} d q=1$ in QMFL is $\theta(\beta) \psi(q)$. The parameter of deformation $\beta$ assumes the value $0<\beta \leq 1 / 4$. Its properties are $|\theta(\beta)|^{2}<1, \lim _{\beta \rightarrow 0}|\theta(\beta)|^{2}=1$ and the relation $|\theta(\beta)|^{2}-|\theta(\beta)|^{4} \approx \beta$ takes place. In such a way the full probability always is less than 1: $p(\beta)=|\theta(\beta)|^{2}=\int|\theta(\beta)|^{2}|\psi(q)|^{2} d q<1$ and it tends to 1 when $\beta \rightarrow 0$. In the most general case of arbitrarily normed state in QMFL $\psi=\psi(\beta, q)=\sum_{n} a_{n} \theta_{n}(\beta) \psi_{n}(q)$ with $\sum_{n}\left|a_{n}\right|^{2}=1$ the full probability is $p(\beta)=\sum_{n}\left|a_{n}\right|^{2}\left|\theta_{n}(\beta)\right|^{2}<1$ and $\lim _{\beta \rightarrow 0} p(\beta)=1$.

It is natural that in QMFL Shrodinger's equation is also deformed. It is replaced by the equation

$$
\frac{\partial \psi(\beta, q)}{\partial t}=\frac{\partial[\theta(\beta) \psi(q)]}{\partial t}=\frac{\partial \theta(\beta)}{\partial t} \psi(q)+\theta(\beta) \frac{\partial \psi(q)}{\partial t},
$$


where the second term in the right side generates the Shrodinger's equation since

$$
\theta(\beta) \frac{\partial \psi(q)}{\partial t}=\frac{-i \theta(\beta)}{\hbar} H \psi(q)
$$

Here $H$ is the Hamiltonian and the first member is added, similarly to the member appearing in the deformed Loiuville's equation and vanishing when $\theta[\beta(t)] \approx$ const. In particular, this takes place in the low energy limit in $\mathrm{QM}$, when $\beta \rightarrow 0$. It follows to note that the above-described theory is not time-reversal as QM, since the combination $\theta(\beta) \psi(q)$ breaks down this property in the deformed Shrodinger's equation. Time-reversal is conserved only in the low energy limit, when quantum mechanical Shrodinger's equation is valid.

\section{Conclusion}

It follows to note, that in some well-known papers on GUR and Quantum Gravity (see for instance [1, 2, 3, 4, 24) there is not any mention about the measuring procedure. However, it is clear that this question is crucial and it cannot be ignored or passed over in silence. We would like to remark, that the measuring rule used in (29], formula (5)) coincide with ours. Taking into account this state of affairs we propose in this paper a detailed treatment of the problem of measurement. In the paper the measuring rule (5) is proposed as a good initial approximation to the exact measuring procedure in QMFL. Corrections to this procedure could be defined by an adequate and fully established description of the space-time foam (see 25]) at Planck's scale. On the other hand, as it was noted in (see [26]) all known approaches dealing with Quantum Gravity one way or another lead to the notion of fundamental length. Involving that notion too, GUR (11) are well described by the inflation model [27]. Therefore, it seems impossible to understand physics on Planck's scale disregarding the notion of fundamental length. One more aspect of this problem should be considered. As it was noted in [28, advancement of a new physical theory implies the introduction of a new parameter and deformation of the precedent theory by this parameter. In essence, all these deformation parameters are fundamental constants: $G, c$ and $\hbar$ (more exactly in [28] $1 / c$ is used instead of $c$ ). As follows from the above results, in the problem from [28] one may redetermine, whether a theory we are seeking is the theory with fundamental length involving these three parameters by definition: 
$L_{p}=\sqrt{\frac{G \hbar}{c^{3}}}$. Notice also that the deformation introduced in this paper is stable in the sense indicated in [28].

The present paper is the continuation and correction of the 33 .

\section{References}

[1] G.Veneziano,A stringly nature needs just two constant Europhys.Lett.2(1986)199;D.Amati,M.Ciafaloni and G.Veneziano,Can spacetime be probed below the string size? Phys.Lett.B216(1989)41; E.Witten, Reflections on the Fate of Spacetime Phys.Today,49(1996)24

[2] R.J.Adler and D.I.Santiago,On Gravity and the Uncertainty Principle, Mod.Phys.Lett.A14(1999)1371 gr-qc/9904026

[3] D.V.Ahluwalia,Wave-Particle duality at the Planck scale: Freezing of neutrino oscillations Phys.Lett. A275 (2000)31, gr-qc/0002005;Interface of Gravitational and Quantum Realms Mod.Phys.Lett. A17(2002)1135, gr-qc/0205121; M.Maggiore, A Generalized Uncertainty Principle in Quantum Gravity Phys.Lett.B304(1993)65, hep-th/9301067

[4] M.Maggiore,Quantum Groups,Gravity and Generalized Uncertainty Principle Phys.Rev.D49(1994)5182, hep-th/9305163; The algebraic structure of the generalized uncertainty principle Phys.Lett.B319(1993)83, hep-th/9309034 ;S.Capozziello,G.Lambiase and G.Scarpetta, The Generalized Uncertainty Principle from Quantum Geometry gr-qc/9910017

[5] W.Heisenberg,Uber den anschaulichen Inhalt der quantentheoretischen Kinematik und Mechanik, Zeitsch.fur Phys,43(1927)172

[6] Y.J.Ng, H.van Dam,Measuring the Foaminess of Space-Time with Gravity-Wave Interferometers,Found.Phys.30(2000)795, gr-qc/9906003

[7] Y.J.Ng, H.van Dam, On Wigner's clock and the detectability space-time foam with gravitational-wave interferometers Phys.Lett.B477(2000)429, gr-qc/9911054 
[8] J.C.Baez, S.J.Olson,Uncertainty in Measurment of Distance, gr-qc/0201030

[9] A.H.Guth,Inflation and EternaL Inflation, astro-ph/0002156

[10] J.A.Wheeler,in Battele Rencontres,ed. by C.DeWitt and J.A. Wheeler (Benjamen,NY,1968)123; B.DeWitt,Quantum Thery Gravity I.The Canonical Theory, Phys.Rev.160(1967)1113.

[11] R.Penrose,Singularities and Asymmetry in Time, in General Relativity, Cambridge University Press, Cambridge, 1979

[12] A.Strominger, Les Houches Lectures on Black Holes, hep-th/9501071

[13] I.B.Khriplovich,R.V. Korkin, How Is the Maximum Entropy of a Quantized Surface Related to Its Area? gr-qc/0112074

[14] S.Hawking,Breakdown of Predictability in Gravitational Collapse, Phys.Rev.D14(1976)2460

[15] S.Giddings, The Black Hole Information Paradox, hep-th/9508151

[16] R.Myers,Pure states don’t wear black, gr-qc/9705065

[17] R.Adler,P.Chen and D.Santiago,The Generalised Uncertainty Principle and Black Hole Remnants,Gen.Rel.Grav.33(2001)2101, gr-qc/0106080; P.Chen and R.Adler, Black Hole Remnants and Dark Matter, gr-qc/0205106

[18] S.Hawking,Particle Creation by Black Holes,Comm.Math.Phys.87(1982)395

[19] R.Penrose,Quantum Theory and Space - Time, in S.Hawking and R.Penrose ,The Nature of Space and Time, Princeton University Press,Princeton,New Jersey,1994

[20] I.B.Khriplovich,Quantization and entropy of black holes, gr-qc/0106012 ;I.B. Khriplovich,Entropy and Area of Black Holes in Loop Quantum Gravity, gr-qc/0109092

[21] S.Hawking, Black Holes and Thermodynamics,Phys.Rev.D13(1976)191 
[22] P.Majumdar,Black hole entropy: classical and quantum aspects, Expanded version of lectures given at the YATI Conference on Black Hole Astrophysics, Kolkata, India, April 2001, hep-th/0110198; S.Das, P.Majumdar and R.K. Bhaduri,General Logarithmic Corrections to Black Hole Entropy, hep-th/0111001;E.C.Vagenas,Quantum Corrections to the Bekenstein-Hawking entropy of the BTZ Black Hole via Self-Gravitation, hep-th/0109108

[23] T.Banks,L.Susskind and M.Peskin,Difficulties for the evolution of pure states into mixed states,Nucl.Phys.B244(1984)125; M.Srednicki,Is purity eternal? hep-th/9206056

[24] S.Hawking, The Unpredictability of Quantum Gravity, Comm.Math.Phys.87(1982)395;S.Hawking,Non-trivial Topologies in Quantum Gravity,Nucl.Phys.B244(1984)135

[25] Wheeler J.A., in: Relativity, Groups and Topology,eds.B.S. and C.M. DeWitt.Gordon and Breach,N.Y.,1963

[26] L.Garay,Quantum Gravity and Minimum Length Int.J.Mod.Phys.A.A10(1995)145 gr-qc/9403008

[27] S.F.Hassan and M.S.Martin, Trans-Plancian Effects in Inflationary Cosmology and Modified Uncertainty Principle, hep-th/0204110

[28] L.Faddeev, Mathematical View on Evolution of Physics, Priroda $5(1989) 11$

[29] A.Kempf,G.Mangano,R.B.Mann,Hilbert Space Representation of the Minimal Length Uncertainty Relation,Phys.Rev.D52(1995)1108hep-th/9412167

[30] A.D.Hefler, Do black holes radiate? gr-qc/0304042

[31] P.S.Custodio,J.E.Horvath,The Generalized Uncertainty Principle,entropy bounds and black hole (non-)evaporation in thermal bath, gr-qc/0305022

[32] J.D.Bekenstein,Black Holes and Entropy,Phys.Rev.D7(1973),2333

[33] A.E.Shalyt-Margolin and J.G.Suarez,Quantum Mechanics of the Early Universe and its Limiting Transition, gr-qc/0302119 